\title{
The impact of sitagliptin, inhibitor of dipeptidyl peptidase-4 (DPP-4), on the ADMA-DDAH-NO pathway in ischemic and reperfused rat livers
}

\author{
Małgorzata Trocha1,A-D,F, Beata Nowak ${ }^{1, C, E}$, Anna Merwid-Ląd ${ }^{1, D}$, Andrzej Szuba ${ }^{2,3, C}$, Piotr Dzięgiel ${ }^{4,5, E}$, Małgorzata Pieśniewskaa, \\ Agnieszka Gomułkiewicz ${ }^{4, B}$, Jerzy Wiśniewski ${ }^{6, C}$, Tomasz Piasecki ${ }^{7, B}$, Magdalena Gziut ${ }^{1, D}$, Adam Szeląg ${ }^{1, E, F}$, Tomasz Sozański ${ }^{1, C, E}$ \\ 1 Department of Pharmacology, Faculty of Medicine, Wroclaw Medical University, Poland \\ 2 Department of Internal Medicine, $4^{\text {th }}$ Military Hospital with Policlinic, Wrocław, Poland \\ ${ }^{3}$ Department of Internal Nursing, Faculty of Health Sciences, Wroclaw Medical University, Poland \\ ${ }^{4}$ Department of Histology and Embryology, Faculty of Medicine, Wroclaw Medical University, Poland \\ ${ }^{5}$ Department of Histology and Embryology, Poznan University of Medical Sciences, Poland \\ ${ }^{6}$ Department of Medical Biochemistry, Faculty of Medicine, Wroclaw Medical University, Poland \\ ${ }^{7}$ Department of Epizootiology and Clinic of Bird and Exotic Animals, Wroclaw University of Environmental and Life Sciences, Poland \\ A - research concept and design; $\mathrm{B}$ - collection and/or assembly of data; $\mathrm{C}$ - data analysis and interpretation; \\ $D$ - writing the article; $E$ - critical revision of the article; $F$ - final approval of the article
}

Address for correspondence

Małgorzata Trocha

E-mail:malgorzata.trocha@umed.wroc.pl

Funding sources

The study was financially supported by Wroclaw

Medical University statutory research funding

(ST-555).

Conflict of interest

None declared

Received on April 24, 2017

Reviewed on May 24, 2017

Accepted on June 25, 2017

DOI

10.17219/acem/75499

Copyright

Copyright by Author(s)

This is an article distributed under the terms of the

Creative Commons Attribution Non-Commercial License

(http://creativecommons.org/licenses/by-nc-nd/4.0/)

\section{Abstract}

Background. A correlation between the level of asymmetric dimethylarginine (ADMA) - the inhibitor of the nitric oxide (NO) synthesis - and the liver function and survival after a liver transplantation has been reported.

Objectives. The aim of this study was to evaluate the effect of sitagliptin - the inhibitor of dipeptidyl peptidase-4 (DPP-4) - on the N0-ADMA-dimethylarginine dimethylaminohydrolase (DDAH) pathway in rat livers subjected to ischemia/reperfusion (IR).

Material and methods. The rats received sitagliptin (5 mg/kg, per os - p.o.) (groups: $S$ - livers not subjected to IR procedure, and SIR - livers subjected to IR procedure) or a saline solution (groups: ( - livers not subjected to IR procedure, and (IR - livers subjected to IR procedure) for 14 days; following this, livers in the SIR and (IR groups were subjected to ischemia (60 min) and reperfusion (24 h). Aminotransferases were measured before the surgery; additionally, the arginine (ARG), ADMA and symmetric dimethylarginine (SDMA) levels were estimated just before ischemia and during reperfusion (at $0.5 \mathrm{~h}, 4 \mathrm{~h}$ and $24 \mathrm{~h}$ ). After $\mathbb{R}$, citrulline, the DDAH activity, mRNA for type 1 DDAH (DDAH1), and arginine methyltransferase type 1 (PRMT1) were determined.

Results. The increase in the initial level of $A R G / A D M A 0(A / A)$ ratio in group $S$ compared to group C verged on statistical significance. At $0.5 \mathrm{~h}$ and $4 \mathrm{~h}$ of reperfusion, the highest concentration of ADMA was found in group CIR. At those time points, the ARG level and the A/A ratio were decreased in groups CIR and SIR as compared to groups ( and S, respectively. The alanine transaminase (ALT) activity was lower in the sitagliptintreated group than in the non-treated one. The DDAH and citrulline levels were reduced in group CIR as compared to group C, but were greater in group SIR as compared to group $S$. The PRMT1 mRNA expression was higher in groups CIR and SIR, compared to groups $C$ and S, respectively.

Conclusions. The increased A/A ratio suggests a protective effect of sitagliptin on livers not subjected to IR. Changes in the DDAH activity and the PRMT1 mRNA expression also imply the protective activity of sitagliptin during $\mathbb{R}$.

Key words: liver, rat, ischemia/reperfusion, asymmetric dimethylarginine, sitagliptin 


\section{Introduction}

Nitric oxide (NO), synthetized from arginine (ARG), is an essential factor regulating relaxation and dilatation of hepatic stellate cells, and hence, sinusoid blood flow. ${ }^{1-3}$ Endothelial NO synthase (eNOS) is responsible for both the basal production of $\mathrm{NO}$ and, when larger amounts of NO are required, the production for its inducible form - iNOS. At a higher concentration, NO reacts with superoxide, forming peroxinitrite, which augments cell injury. ${ }^{3,4}$ Numerous individual factors influence the NO concentration, including the levels of eNOS cofactors - the reduced form of nicotinamide adenine dinucleotide phosphate (NADPH) and tetrahydropterin, oxygen support, the ARG level, the argininase activity, and the concentration of methylated derivatives of ARG, including asymmetric and symmetric dimethylarginine (ADMA, SDMA) and N-monomethylL-arginine (L-NMMA), known as endogenous inhibitors of NOS. The regulation of the ADMA synthesis by arginine methyltransferases (PRMTs), and its degradation by dimethylarginine dimethylaminohydrolase (DDAH) to dimethylamine and citrulline are also highly important. ${ }^{5}$

Liver injury during a partial hepatectomy or a transplantation is initially caused by ischemia and aggravated by reperfusion of hepatic parenchyma. Generally, reperfusion can be divided into 2 phases. During the first 3-6 h of renewed blood flow, the generation of reactive oxygen species (ROS) and NO, and the activation of T cells and Kupffer cells can be observed; following this, the neutrophil infiltration, leading to the cytokine, chemokine and ROS production, is seen within the next 18-24 h. ${ }^{6,7}$ Asymmetric dimethylarginine, released during reperfusion, is one of the factors inhibiting the synthesis of NO. Vasoconstriction, considered a consequence of the imbalance between $\mathrm{NO}$ and endothelin, has been postulated to form the basis of disturbed liver function in ischemia/reperfusion (IR). ${ }^{8,9}$ Notwithstanding the upregulated protein degradation with the relaxation of free methylarginines, impaired degradation of the latter has also been observed in such conditions.Furthermore, as the correlation between the level of ADMA and the liver function and survival after a liver transplantation has been reported, the identification of substances decreasing the ADMA level and the elucidation of the mechanism of their influence on the NO level appears crucial. ${ }^{5}$

Sitagliptin is an oral hypoglycemic drug proficient in inhibiting dipeptidyl peptidase-4 (DPP-4) - an enzyme involved in the metabolism of incretins, such as glucagonlike peptide-1 (GLP-1). ${ }^{10}$ Although sitagliptin is a relatively new drug, introduced to European Pharmacopeia in 2007, individual cases of its antioxidative and anti-inflammatory action have been reported, including the inhibition of the DPP-4 activity, which reduced IR injury in mice after a lung transplantation. ${ }^{11-13}$

The aim of the current work was to investigate the effect of sitagliptin on ARG and its derivatives as well as the activity of DDAH, DDAH1 and PRMT1 mRNA - enzymes involved in the production and degradation of ADMA, in rat livers subjected and not subjected to IR. The experiment was carried out on healthy rodents to eliminate the influence of harmful factors, extraneous to IR, on the liver function.

\section{Material and methods}

\section{Animals}

Thirty-six Wistar male rats were included in this study. Animals were housed in individual chambers with a 12:12 h light-dark cycle. The temperature was maintained at $21-23^{\circ} \mathrm{C}$. The experiment protocol was approved by the Local Ethics Commission for Experiments on Animals (No. 80/2012 of December 5, 2012).

\section{Chemicals}

Sitagliptin (Januvia - tablets, 100 mg; MSD sp. z o.o., Warszawa, Poland), heparin (Heparinum WZF - ampoules, 25,000 U/5 mL; Polfa Warszawa S.A., Warszawa, Poland), ketamine hydrochloride (Bioketan; Vetoquinol Biowet, Gorzów Wielkopolski, Poland), medetomidine hydrochloride (Domitor - ampoules, $1 \mathrm{mg} / \mathrm{mL}$; Orion Pharma, Espoo, Finland), 0.9\% sodium chloride solution (Polpharma S.A., Starogard Gdański, Poland), and Ringer's solution (Polfa Lublin S.A., Lublin, Poland) were used in the study.

\section{Experimental design}

Following acclimatization, animals were divided randomly into 4 groups: groups $C(n=9)$ and $S(n=8)-$ the livers were not subjected to IR procedure, and groups CIR $(n=9)$ and SIR $(n=10)$ - the livers undergoing IR procedure. Rat livers from groups $\mathrm{S}$ and SIR received sitagliptin intragastrically $(5 \mathrm{mg} / \mathrm{kg}$ ) once a day for 14 days, prior to the surgical procedure. To determine the initial levels of ARG derivatives and activities of aminotransferases - alanine transaminase (ALT) and aspartate transaminase (AST) - blood samples were obtained from the tail vein after the drug treatment.

\section{Preparation of the liver ischemia/reperfusion injury model}

Following the administration of medetomidine $(0.1 \mathrm{mg} /$ $\mathrm{kg}$ intramuscularly - i.m.) and ketamine (7 mg/kg i.m.) anesthesia, the animals underwent a midline laparotomy. In the CIR and SIR groups, the ischemia of median and left lateral liver lobes was achieved by occluding the branches of the portal vein and hepatic artery with a microvascular clip. After ischemia (60 min), the clip was removed and reperfusion was started $(24 \mathrm{~h})$. At $0.5 \mathrm{~h}, 4 \mathrm{~h}$ and $24 \mathrm{~h}$ 
of reperfusion, samples of blood were collected to mark the ADMA, SDMA and ARG levels. The citrulline concentration was measured at $24 \mathrm{~h}$ of reperfusion. Once the experiment was terminated, the livers were weighted and ischemic lobes were isolated. In the $C$ and $S$ groups, the animals underwent the same surgical procedure as in the ischemic groups, but after a laparotomy, the branches of the portal vein and hepatic artery were not occluded.

A portion of the obtained liver lobes was placed in the RNAlater RNA Stabilization Reagent (Qiagen, Hilden, Germany) and used for the real-time polymerase chain reaction (PCR); the remaining liver tissues were homogenized and the supernatant was collected. ${ }^{14}$

\section{Blood enzymes, arginine and its derivatives, and citrulline}

The activity of DDAH was determined spectrophotometrically (Marcel S350 PRO; Marcel sp. z o.o., Zielonka, Poland), using the method based on the L-citrulline production rate, described in our previous study. ${ }^{15}$ Briefly, $1 \mathrm{mM}$ of ADMA was added to each sample of the homogenate with a phosphate buffer ( $\mathrm{pH}$ 6.5), and then incubated for $45 \mathrm{~min}$ at $37^{\circ} \mathrm{C}$. The reaction was stopped with $4 \%$ sulfosalicylic acid and the samples were centrifuged. Following this, diacetic monooxime in $5 \%$ acetic acid mixed with antipyrine in $50 \%$ sulfuric acid was added to the samples. L-citrulline was determined following the incubation at $60^{\circ} \mathrm{C}$ and cooling on ice. The DDAH activity was presented as $\mu \mathrm{m}$ of L-citrulline/gram of protein $/ \mathrm{min}$ at $37^{\circ} \mathrm{C}$.

The concentrations of amide derivative ARG, citrulline, ADMA, and SDMA were measured simultaneously by highperformance liquid chromatography (HPLC) with mass spectrometry detection. ${ }^{16}$ All the separations were performed with the HPLC system Agilent Technologies 1260 Infinity (Agilent Technologies, Santa Clara, USA). The Acquity UPLC HSS T3 $50 \times 1.0 \mathrm{~mm}, 1.8 \mu \mathrm{m}$ column from Waters (Milliford, USA) was used throughout the chromatographic separation. A 0.22-micrometer membrane inline filter from Waters was installed to protect the analytical column. The volume of injection was $2 \mu \mathrm{L}$. Gradient elution was performed using $0.1 \%$ formic acid in water $(\mathrm{A})$ and $0.1 \%$ formic acid in methanol (B) at a flow rate of $0.2 \mathrm{~mL} / \mathrm{min}$. The overall time of the method was $14.5 \mathrm{~min}$ and consisted of 7 steps: from $0 \mathrm{~min}$ to $1 \mathrm{~min}, 10 \% \mathrm{~B}$; from $1 \mathrm{~min}$ to $6 \mathrm{~min}$, $10-35 \% \mathrm{~B}$; from $6 \mathrm{~min}$ to $7 \mathrm{~min}, 35-60 \% \mathrm{~B}$; from $7 \mathrm{~min}$ to $7.5 \mathrm{~min}, 60-90 \% \mathrm{~B}$; from $8 \mathrm{~min}$ to $8.5 \mathrm{~min}, 90-100 \% \mathrm{~B}$. All the separations were conducted at $60^{\circ} \mathrm{C}$.

The level of protein in liver homogenates and the activity of aminotransferases in serum were determined in a certified laboratory.

\section{Real-time polymerase chain reaction}

Total RNA was extracted from the studied tissues using the RNeasy Mini Kit (Qiagen) as described in the protocol.
Reverse transcription of mRNA to cDNA was performed using the QuantiTect Reverse Transcription Kit (Qiagen). The expression of DDAH1 and PRMT1 mRNA was evaluated by the real-time PCR in the 7900HT Fast Real-Time PCR System (Applied Biosystems, Carlsbad, USA). Glyceraldehyde-3-phosphate dehydrogenase (GAPDH) was used as a reference gene. The real-time PCR was performed using the TaqMan Gene Expression Master Mix, and specific primers and TaqMan probes: Rn 00574200_m1 for DDAH1, Rn 00821202_g1 for PRMT1 and Rn 99999916 _ $\mathrm{m} 1$ for $G A P D H$ (Applied Biosystems). All the reactions were performed in triplicates in standardized thermal cycling conditions, including the polymerase activation at $50^{\circ} \mathrm{C}$ for $2 \mathrm{~min}$, denaturation at $94^{\circ} \mathrm{C}$ for $10 \mathrm{~min}$ and 40 cycles of denaturation at $94^{\circ} \mathrm{C}$ for $15 \mathrm{~s}$, followed by annealing and synthesis at $60^{\circ} \mathrm{C}$ for $1 \mathrm{~min}$. Relative quantity (RQ) of DDAH1 and PRMT1 mRNA was calculated using the 2- $\Delta \Delta \mathrm{Ct}$ method.

\section{Statistical analysis}

Data was expressed as mean \pm standard deviation (SD). The two-way analysis of variance (ANOVA) was used to analyze the impact of sitagliptin and IR on the activity of DDAH and the mRNA expression for DDAH and PRMT. The one-way ANOVA was used to determine the effect of the sitagliptin administration on the initial values of aminotransferases. The analysis of variance with repeats was used for the statistical analysis of the influence of the drug and the time of reperfusion on the ARG, ADMA and SDMA levels, and the ARG/ADMA (A/A) ratio. Specific comparisons were made with the contrast analysis. Hypotheses were considered positively verified at $\mathrm{p}<0.05$.

\section{Results}

\section{Asymmetric dimethylarginine, symmetric dimethylarginine, arginine, and the arginine/ /asymmetric dimethylarginine ratio}

After the sitagliptin administration, no significant differences between all the groups in the ADMA0, ARG0 and SDMA0 levels were found (Fig. 1). The observed increase in the A/A0 ratio in group $\mathrm{S}$, as compared to group $\mathrm{C}$, was not statistically significant, but it was close to the significance threshold ( $\mathrm{S}$ vs $\mathrm{C} ; \mathrm{p}=0.07$ ).

At $0.5 \mathrm{~h}$ and $4 \mathrm{~h}$ of reperfusion, the highest ADMA concentration was found in group CIR and the lowest in group $\mathrm{S}$ (after $0.5 \mathrm{~h}$ : CIR0.5 vs S0.5; p < 0.05, and after $4 \mathrm{~h}$ : CIR4 vs $\mathrm{S} 4 ; \mathrm{p}<0.01)$. Similar differences were noted in the SDMA levels after $0.5 \mathrm{~h}$ of reperfusion (CIR0.5 vs S0.5; p < 0.05). After $4 \mathrm{~h}$ of reperfusion, the ADMA level was also significantly higher in groups that underwent IR (CIR4 vs C4 and SIR4 v. S4; p < 0.05 in both comparisons) (Fig. 1A,1B).

At $0.5 \mathrm{~h}$ and $4 \mathrm{~h}$ of reperfusion, both the level of ARG and the A/A ratio were the highest in group $\mathrm{S}$ and the 

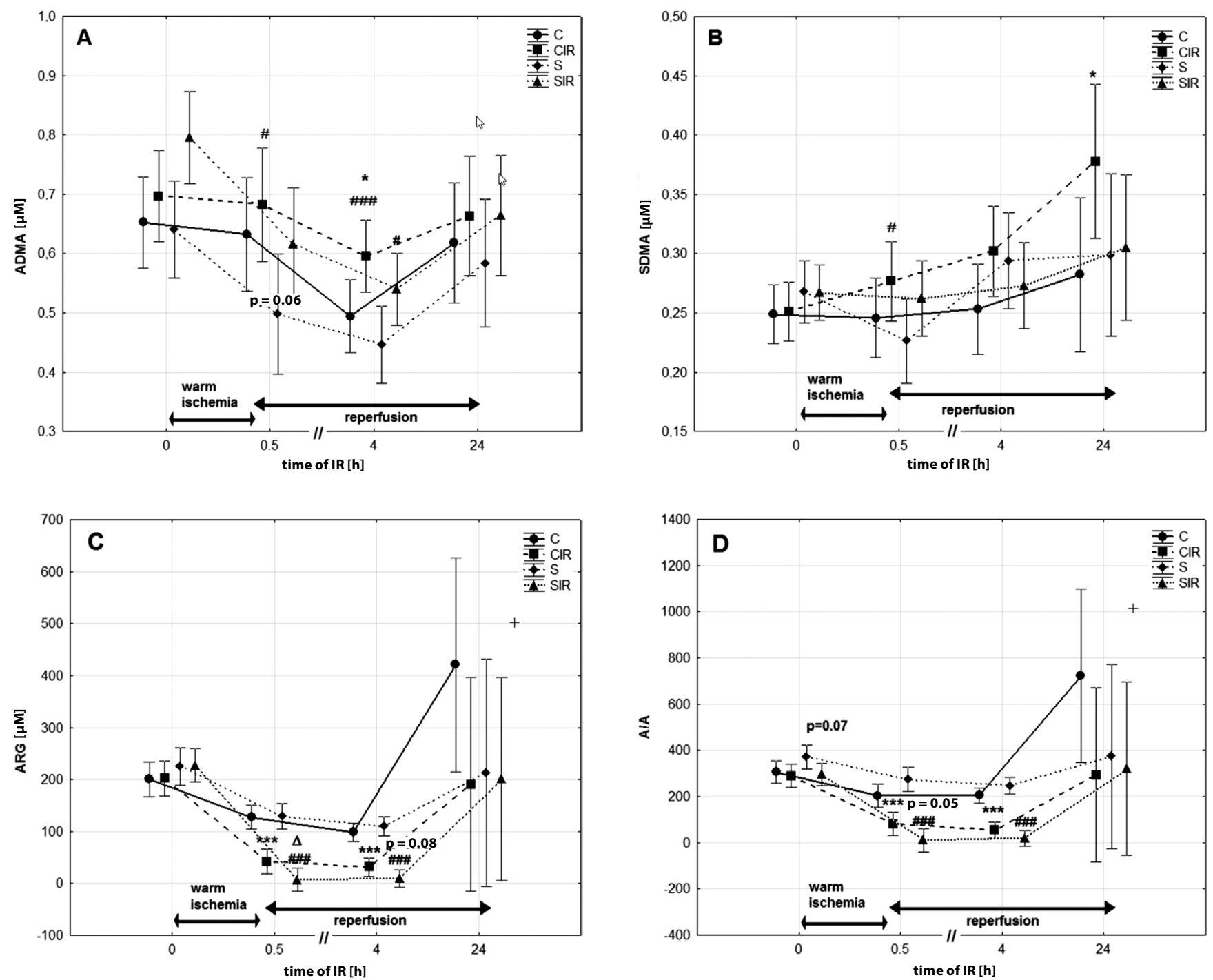

Fig. 1. The influence of IR and the sitagliptin treatment on the levels of $A D M A(A), S D M A(B), A R G(C)$, and the $A / A$ ratio (D)

Values are presented as mean \pm standard deviation (SD); ADMA - asymmetric dimethylarginine; SDMA - symmetric dimethylarginine; A/A - arginine/ asymmetric dimethylarginine; group C - non-treated and not subjected to IR; group CIR - non-treated and subjected to IR; group $S$ - sitagliptin-treated and not subjected to IR; group SIR - sitagliptin-treated and subjected to IR; IR - ischemia/reperfusion; specific comparisons: ${ }^{*} p<0.05$ and ${ }^{* * *} p<0.005$ (compared to C); ${ }^{\#} \mathrm{p}<0.05$ and ${ }^{\# \# \#} \mathrm{p}<0.005$ (compared to S); ${ }^{\Delta} \mathrm{p}<0.05$ (compared to CIR).

lowest in group SIR. Furthermore, at those reperfusion time points, in groups CIR and SIR, the concentration of ARG was significantly lower as compared to groups C and S, respectively (CIR0.5 vs C0.5 and SIR0.5 vs S0.5, as well as CIR4 vs C4 and SIR4 vs S4; p $<0.005$ in all comparisons). Similarly, the level of the A/A ratio was also significantly lower in groups that underwent IR (CIR0.5 vs C0.5 and SIR0.5 vs S0.5, as well as CIR4 vs C4 and SIR4 vs S4; p $<0.005$ in all comparisons). At $0.5 \mathrm{~h}$ of reperfusion, the concentration of ARG was significantly lower in group SIR than in group CIR (SIR0.5 vs CIR0.5; $\mathrm{p}<0.05)$. The level of the A/A ratio did not reach significance, but it was close to the significance threshold (SIR0.5 vs CIR0.5; p = 0.05). Similarly, at $4 \mathrm{~h}$ of reperfusion, the difference in the concentration of ARG was near the threshold of statistical significance (SIR4 vs CIR4; $\mathrm{p}=0.08)($ Fig. 1C,1D)
At $24 \mathrm{~h}$ of reperfusion, the levels of the 3 examined parameters (ADMA, ARG and the A/A ratio) were restored to baseline values. At this time, SDMA was significantly higher in group CIR than in group C (CIR24 vs C24; $\mathrm{p}<0.05$ ) (Fig. 1).

\section{Biochemical analyses}

The activity of dimethylarginine dimethylaminohydrolase

The activity of DDAH in liver homogenates was lower in group $S$ as compared to group $C$ ( $S$ vs $C$; $p<0.005$ ). In IR conditions, the DDAH activity decreased in group CIR as compared to group C (CIR vs C; $\mathrm{p}<0.01)$; in contrast, in drug-treated groups, it was significantly increased in group SIR compared to group S (SIR vs S; p < 0.05) (Fig. 2A). 
A activity of DDAH [ $\mu \mathrm{M}$ of citrulline]

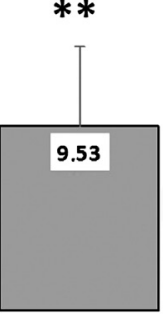

CIR

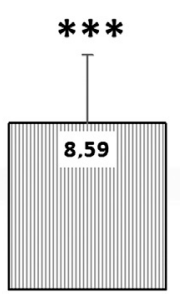

S
B

the concentration of citrulline $[\mu \mathrm{M}]$

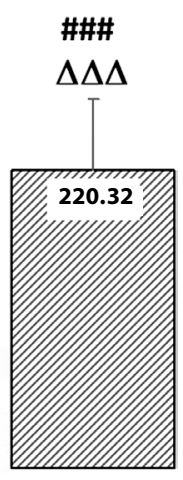

SIR

Fig. 2. The influence of $I R$ and the sitagliptin treatment on the DDAH activity $(A)$ and the citrulline concentration (B)

Values are presented as mean \pm standard deviation (SD); DDAH - dimethylarginine dimethylaminohydrolase; group C - non-treated and not subjected to IR; group CIR - non-treated and subjected to IR; group S - sitagliptin-treated and not subjected to IR; group SIR - sitagliptin-treated and subjected to IR; IR - ischemia/reperfusion; specific comparisons: ${ }^{*} p<0.05$, ** $p<0.01$, ${ }^{* * *} p<0.005$ (compared to C); $p<0.05$ and ${ }^{\# \# \# ~} p<0.005$ (compared to S);

${ }^{\Delta \Delta \Delta} \mathrm{p}<0.005$ (compared to CIR).

\section{Citrulline}

After $24 \mathrm{~h}$ of reperfusion, the concentration of citrulline decreased in group CIR compared to group C (CIR vs C; $\mathrm{p}<0.05$ ); however, in drug-treated groups, it was significantly increased in group SIR as compared to groups $S$ and CIR (SIR vs S and SIR vs CIR; $\mathrm{p}<0.005$ ) (Fig. 2B).

\section{Aminotransferases}

After 2 weeks of the sitagliptin administration, the activity of ALT was significantly lower in group $S$ than in group $\mathrm{C}$ ( $\mathrm{S}$ vs $\mathrm{C} ; \mathrm{p}<0.05)$. No significant changes in the AST activity were observed in all the examined groups (Table 1).

The dimethylarginine dimethylaminohydrolase and arginine methyltransferase mRNA expression

The DDAH1 mRNA expression was observed to be the lowest in group $S$ and the highest in group $C$, but the differences were not statistically significant. The PRMT1 mRNA expression was higher in groups CIR and SIR as compared to groups $C$ and $S$, respectively (CIR vs $C$ and SIR vs S; $\mathrm{p}<0.005$ ) (Fig. 3A,3B).

A mRNA for DDAH type 1

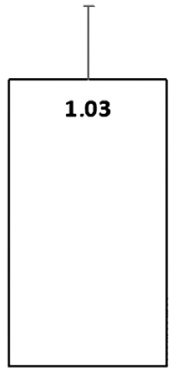

C

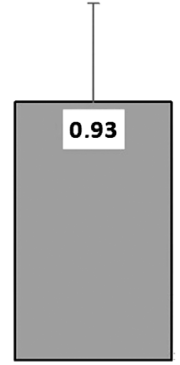

CIR

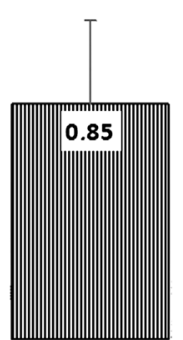

S

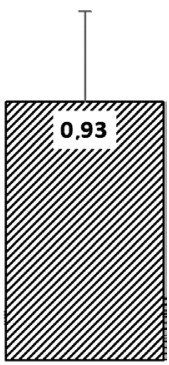

SIR
Table 1. The values of the ALT and AST activity after 2 weeks of treatment with sitagliptin at a dose of $5 \mathrm{mg} / \mathrm{kg}$ intragastrically (groups $\mathrm{S}$ and SIR) or with a saline solution (groups $C$ and CIR)

\begin{tabular}{|l|c|c|}
\multicolumn{1}{|c}{ Groups } & ALT [U/L] & AST [U/L] \\
\cline { 2 - 3 } \multicolumn{1}{|c|}{} & mean \pm SD & mean \pm SD \\
\hline C $(n=9)$ & $66.89 \pm 20.14$ & $175.11 \pm 104.08$ \\
\hline CIR $(n=9)$ & $88.67 \pm 55.14$ & $178.78 \pm 93.88$ \\
\hline$S(n=8)$ & $46.00^{*} \pm 8.09$ & $206.75 \pm 51.87$ \\
\hline $\operatorname{SIR}(n=10)$ & $62.80 \pm 10.61$ & $186.90 \pm 26.54$ \\
\hline
\end{tabular}

IR - ischemia/reperfusion; ALT - alanine transaminase; AST - aspartate transaminase; SD - standard deviation; ${ }^{*} \mathrm{p}<0.05$ (compared to C).

\section{Discussion}

Despite significant progress in available therapies, an organ transplantation often remains the only option of treatment for many patients. An important limitation of this method is the damage suffered by the transplanted organs following ischemia, and particularly during the

B

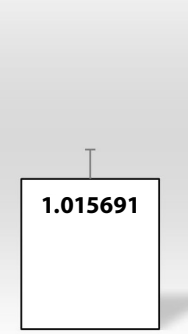

C

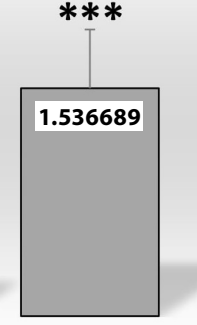

$\mathrm{CIR}$
mRNA for PRMT type 1

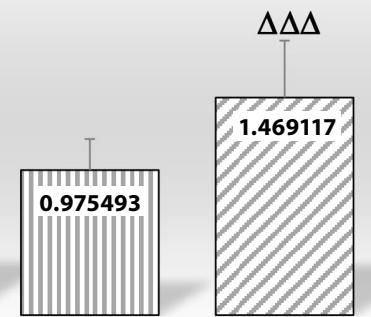

SIR

Fig. 3. The influence of IR and the sitagliptin treatment on the relative expression of PRMT1 (A) and DDAH1 (B) mRNA

Data is expressed as a percentage of control values and as mean \pm standard deviation (SD); PRMT1 - arginine methyltransferase type 1;

DDAH1 - dimethylarginine dimethylaminohydrolase type 1; group C - non-treated and not subjected to IR; group CIR - non-treated and subjected to IR; group S - sitagliptin-treated and not subjected to IR; group SIR - sitagliptin-treated and subjected to IR; IR - ischemia/reperfusion; specific comparisons: ${ }^{* * *} p<0.005$ (compared to C); ${ }^{\Delta \Delta \Delta} p<0.005$ (compared to S). 
subsequent reperfusion. Consequently, numerous studies have focused on the elucidation of novel substances with a protective activity under these conditions, or novel, advantageous properties of already known, chronically administrated substances. For that reason, the present study set out to clarify the activity of hypoglycemic drug - sitagliptin - in a liver undergoing IR. The key conclusions are as follows:

- in the group treated with sitagliptin, not subjected to IR, an increase in the level of the A/A ratio measured just before the surgery, as compared to the untreated group, verged on statistical significance, the DDAH activity was significantly lowered and the mRNA expression for $D D A H 1$ was slightly lowered as compared to the untreated group, while the ALT activity was significantly lower in the drug-treated group than in the untreated group;

- in the drug-treated and subjected to IR group, the ARG level and the A/A ratio were significantly decreased compared to the group non-subjected to IR and also to the untreated group subjected to IR, the ADMA level was higher as compared to the treated group without IR, while the levels of DDAH and citrulline were higher than in the drug-treated non-IR group;

- in the untreated groups subjected to IR, the ADMA and SDMA concentrations measured during reperfusion were the highest among all the groups, the ARG level and the A/A ratio were significantly decreased as compared to the group non-subjected to IR, the levels of DDAH and citrulline were increased and the PRMT1 mRNA expression was increased as compared to the group without IR.

Asymmetric dimethylarginine inhibits the activity of all isoforms of NOS. Symmetric dimethylarginine does not have a direct impact on NO synthases, but may also downregulate their activity, acting as a competitor of the ARG transport through the cell membrane by the cationic amino acid transporter (CAT) y+ system. ${ }^{17}$ In various pathological conditions (e.g., hypercholesterolemia, hyperglycemia, hyperhomocysteinemia, arterial hypertension, diabetes, and cardiac failure), the ADMA and SDMA levels increase, causing a reduction in the NO production and the dysfunction of endothelium. ${ }^{4,18}$ Increased ADMA levels are also associated with liver damage during IR. ${ }^{8}$ In patients with severely impaired liver function after a major hepatic resection, an increased level of ADMA was observed during the postoperative period. ${ }^{19}$ In our work, the ADMA level during reperfusion was the highest in the untreated group subjected to IR, and was significantly higher than in the non-IR group. At $0.5 \mathrm{~h}$ of reperfusion, the SDMA level was the highest, which may be an additional reason for the inhibition of the NO production. Following $24 \mathrm{~h}$ of reperfusion, the concentrations of both methylarginines were similar in all the groups, which indicated that the observed changes were relatively reversible and that monitoring changes in those parameters during the experiment was more valuable than a single measurement at the end of reperfusion.
The level of ARG, being the main substrate for NOS, may also influence the bioavailability of NO. ${ }^{20}$ In the case of ARG deficiency, eNOS assumes the uncoupled form and catalyzes a reaction with molecular oxygen instead of ARG, leading to the production of peroxide instead of NO. ${ }^{21}$ The balance between the ADMA and ARG level is crucial and the ARG activity depends on the initial level of ADMA. In the case of hypercholesterolemia, the normalization of the endothelium-dependent relaxation after the ARG administration was observed only in patients with increased ADMA levels. ${ }^{22,23}$ As reported by other researchers, and in accordance with our previous experiments, in both ischemic groups, the ARG level and the A/A ratio decreased during reperfusion. ${ }^{24-26}$

Asymmetric dimethylarginine is metabolized to citrulline and dimethylamine in a reaction catalyzed by DDAH. ${ }^{4}$ Therefore, the reduced DDAH activity in the untreated group subjected to IR may be at least partially responsible for increased ADMA levels in our experiment. The citrulline concentration was significantly lowered, in line with the lowered DDAH activity. In pathological conditions, such as stress, ischemic heart disease, diabetes, fasting, or infections, an increased concentration of ADMA may also be a result of increased protein degradation. ${ }^{27,28}$ Under IR injury, the protein methylation is upregulated to remove altered proteins. ${ }^{27}$ The dimethylation of ARG in an asymmetric and symmetric configuration is a reaction catalyzed by PRMTs. ${ }^{29}$ It has been reported that under conditions of hypertension or diabetes, the expression of PRMT1 mRNA increases. ${ }^{30} \mathrm{We}$ also observed an increased mRNA expression for PRMT1 in ischemic livers, which suggests extensive protein degradation under IR. In order to deepen this knowledge, our work should be complemented by a determination of the PRMT activity and its enzymatic protein level.

In rats not subjected to IR, the protective effect of sitagliptin could be seen in terms of the A/A ratio; when measured just before the surgery, it was higher in the treated group. The protective effect of sitagliptin may be, as in other studies, more evident in rats with diabetes than in healthy animals. ${ }^{11,12}$ The ADMA level measured during the surgery was lower in the drug-treated non-ischemic group than in the untreated one. Similarly to the response to a decreased ADMA level, the DDAH activity after the surgery was lower in the drug-treated group compared to the untreated one, which was confirmed by a slightly lowered expression of DDAH1 mRNA. This observation may suggest that sitagliptin is able to protect livers subjected to some degree of gentle manipulation during the surgery.

Based on previous experiments, in which the inhibition of the DPP-4 activity significantly reduced IR injury, ${ }^{31,32}$ and also on literature data suggesting that sitagliptin protects the kidneys from IR injury mainly by inhibiting oxidative stress and the inflammatory reaction, we expected evident effects of sitagliptin on rat livers in IR 
conditions. ${ }^{11,12}$ The DDAH activity and the citrulline concentration significantly increased and were higher as compared to the non-ischemic group, probably as a response to a higher concentration of ADMA. The ARG level and the A/A ratio were significantly lower than in the nonischemic group. The latter parameters were also lower than in untreated rats subjected to IR. This unexpected effect may question the protective effect of sitagliptin in IR conditions. The phenomenon may be explained by a need to reduce the concentration of NO, produced in large quantities under IR, and to augment cell injury. ${ }^{33}$ In other works, sitagliptin or exenatide lowered an increased level of NO in diabetic rats undergoing renal IR. ${ }^{11,34}$ Certainly, this issue needs to be clarified in further studies.

Only a few cases of hepatotoxicity of sitagliptin have been reported so far. ${ }^{35,36}$ Conversely, reports on the beneficial effects of this drug on the liver, reflected by a slight decrease in transaminases, have also been published. ${ }^{37,38}$ Sitagliptin or exenatide exerted a protective effect on the livers also in animals with IR renal injury. ${ }^{11,34}$ In our research, no harmful effects of sitagliptin on the liver function were observed. Although the period of drug administration was rather short and the drug was given in small doses, the results suggest that rat livers were rather improved after the treatment, which was reflected by the lower ALT activity in the group receiving sitagliptin than in the untreated one.

The use of a partial liver ischemia model is one of the limitations of this work. The principal disadvantage is increased blood flow in non-ischemic part of the liver during induced ischemia of the median and left lateral lobe. Due to the collateral blood supply, the exchange of oxygen from the perfused to the ischemic part of the liver may take place. Therefore, differences in the examined parameters were not so evident. It is also worth noting that the livers used in this experiment were derived from healthy animals, and to our knowledge, the effect of sitagliptin in healthy animals is different from that in hypoglycemic ones. However, hyperglycemia is an additional factor contributing to the NO system and the results attained in the work on rats with induced diabetes are more evident. It is also difficult to compare the results obtained in healthy animals to those in diabetic ones.

In conclusion, a slight protective effect of sitagliptin on the ADMA-DDAH-NO pathway in rat livers not subjected to IR was shown in our study. A more evident impact was seen under IR conditions. Probably, the protective effect of sitagliptin is at least partly independent of the main hypoglycemic action of the drug - a fact that we also tried to demonstrate in this work. The results of the present work should be confirmed in future studies, involving other key factors taking part in the development of IR injury. The mechanism of action of sitagliptin during IR may be more complex and may be also associated with anti-inflammatory and antioxidant properties of the drug. ${ }^{11,34}$

\section{References}

1. Maslak E, Gregorius A, Chlopicki S. Liver sinusoidal endothelial cells (LSECs) function and NAFLD: NO-based therapy targeted to the liver. Pharmacol Rep. 2015;67(4):689-694.

2. Langer DA, Shah VA. A gas, an amino acid, and an imposter: The story of nitric oxide, L-arginine, and ADMA in portal hypertension. Hepatology. 2005;42(6):1255-1257.

3. Mòdol T, Natal C, Pérez de Obanos MP, et al. Apoptosis of hepatic stellate cells mediated by specific protein nitration. Biochem Pharmacol. 2011;81(3):451-458.

4. Buttery LD, Springall DR, Chester AH, et al. Inducible nitric oxide synthase is present within human atherosclerotic lesions and promotes the formation and activity of peroxynitrite. Lab Invest. 1996; 75(1):77-85.

5. Tran CT, Leiper JM, Vallance P. The DDAH/ADMA/NOS pathway. Atheroscler Suppl. 2003;4(4):33-40.

6. Fan C, Zwacka RM, Engelhardt JF. Therapeutic approaches for ischemia/reperfusion injury in the liver. J Mol Med. 1999;77(8):577-596.

7. Hines IN, Harada H, Flores S, Gao B, McCord JM, Grisham MB. Endothelial nitric oxide synthase protects the post-ischemic liver: Potential interactions with superoxide. Biomed Pharmacother. 2005;59(4): 183-189.

8. Martin-Sanz P, Olmedilla L, Dulin E, et al. Presence of methylated arginine derivatives in orthotopic human liver transplantation: Relevance for liver function. Liver Transpl. 2003;9(1):40-48.

9. Vallance P, Leiper J. Cardiovascular biology of the asymmetric dimethylarginine: Dimethylarginine dimethylaminohydrolase pathway. Arterioscler Thromb Vasc Biol. 2004;24(6):1023-1030.

10. Deacon CF. Dipeptidyl peptidase 4 inhibition with sitagliptin: A new therapy for type 2 diabetes. Expert Opin Investig Drugs. 2007;16(4): 533-545.

11. Vaghasiya J, Sheth N, Bhalodia Y, Manek R. Sitagliptin protects renal ischemia reperfusion induced renal damage in diabetes. Regulatory Peptides. 2011;166(1-3):48-54.

12. Chen YT, Tsai TH, Yang CC, et al. Exendin-4 and sitagliptin protect kidney from ischemia-reperfusion injury through suppressing oxidative stress and inflammatory reaction. J Trans/ Med. 2013;11:270-289.

13. Wolfgang J, Ingrid DM, Veerle $M$, et al. Inhibition of CD26/DPP IV attenuates ischemia/reprfuson injury in orthotopic mouse lung transplants: The pivotal role of vasoactive intestinal peptide. Peptides. 2010;31:585-591.

14. Morales Al, Vicente-Sanchez C, Jerkic M, et al. Effect of quercetin on metallothionein, nitric oxide synthase and cyclooxygenase-2 expression on experimental chronic cadmium nephrotoxicity in rats. Toxicol Appl Pharmacol. 2006;210(1-2):128-135.

15. Trocha M, Merwid-Ląd A, Chlebda-Sieragowska E, et al. Age-related changes in ADMA-DDAH NO pathway in rat liver subjected to partial ischemia followed by global reperfusion. Exp Gerontol. 2014;50:45-51.

16. Wiśniewski J, Fleszar MG, Piechowicz J, et al. A novel mass spectrometry-based method for simultaneous determination of asymmetric and symmetric dimethylarginine, I-arginine and I-citrulline optimized for LC-MS-TOF and LC-MS/MS. Biomed Chromatogr. 2017;31:1-11. doi: 10.1002/bmc.3994

17. Bode-Böger SM, Scalera F, Kielstein JT, et al. Symmetrical dimethylarginine: A new combined parameter for renal function and extent of coronary artery disease. J Am Soc Nephrol. 2006;17(4):1128-1134.

18. Gore MO, Lüneburg N, Schwedhelm E, et al. Symmetrical dimethylarginine predicts mortality in the general population: Observations from the Dallas heart study. Arterioscler Thromb Vasc Biol. 2013; 33(11):2682-2688.

19. Nijveldt RJ, Teerlink T, Siroen MP, et al. Elevation of asymmetric dimethylarginine (ADMA) in patients developing hepatic failure after major hepatectomy. JPEN J Parenter Enteral Nutr. 2004;28(6):382-387.

20. Lorin J, Zeller M, Guilland JC, Cottin Y, Vergely C, Rochette L. Arginine and nitric oxide synthase: Regulatory mechanisms and cardiovascular aspects. Mol Nutr Food Res. 2014;58(1):101-116.

21. Nguyen MC, Park JT, Jeon YG, et al. Arginase inhibition restores peroxynitrite-induced endothelial dysfunction via L-arginine-dependent endothelial nitric oxide synthase phosphorylation. Yonsei Med J. 2016;57(6):1329-1338.

22. Pope AJ, Karuppiah K, Cardounel AJ. Role of the PRMT-DDAH-ADMA axis in the regulation of endothelial nitric oxide production. Pharmacol Res. 2009;60(6):461-465. 
23. Hornig B, Arakawa N, Böger RH, Bode-Böger SM, Frölich JC, Drexler $\mathrm{H}$. Plasma levels of ADMA are increased and inversely related to endothelium-mediated vasodilation in patients with chronic heart failure: A new predictor of endothelial dysfunction? Circulation. 1998; 98(Suppl 1):318.

24. Dimitrow PP, Undas A, Bober M, Tracz W, Dubiel JS. Plasma biomarkers of endothelial dysfunction in patients with hypertrophic cardiomyopathy. Pharmacol Rep. 2007;59(6):715-720.

25. Trocha M, Merwid-Ląd A, Sozański T, et al. Influence of ezetimibe on ADMA-DDAH-NO pathway in rat liver subjected to partial ischemia followed by global reperfusion. Pharmacol Rep. 2013;65(1): 122-133.

26. Trocha M, Merwid-Ląd A, Szuba A, et al. Effect of simvastatin on nitric oxide synthases (eNOS, iNOS) and arginine and its derivatives (ADMA, SDMA) in ischemia/reperfusion injury in rat liver. Pharmacol Rep. 2010;62(2):343-351.

27. Tran CT, Leiper JM, Vallance P. The DDAH/ADMA/NOS pathway. Atheroscler Suppl. 2003;4(4):33-40.

28. Ferrigno A, Di Pasqua LG, Berardo C, Richelmi P, Vairetti M. Liver plays a central role in asymmetric dimethylarginine-mediated organ injury. World J Gastroenterol. 2015;21(17):5131-5137.

29. Maas R. Pharmacotherapies and their influence on asymmetric dimethylargine (ADMA). Vasc Med. 2005;10(Suppl 1):S49-S57.

30. Matsuguma K, Ueda S, Yamagishi S, et al. Molecular mechanism for elevation of asymmetric dimethylarginine and its role for hypertension in chronic kidney disease. J Am Soc Nephrol. 2006;17(8): 2176-2183.
31. Zhai W, Cardell M, De Meester I, et al. Ischemia/reperfusion injury: The role of CD 28/dipeptidyl-peptidase-IV-inhibition in lung transplantation. Transplant Proc. 2006;38(10):3369-3371.

32. Chang MW, Chen $\mathrm{CH}$, Chen YC, at al. Sitagliptin protects rat kidneys from acute ischemia-reperfusion injury via upregulation of GLP-1 and GLP-1 receptors. Acta Pharmacol Sin. 2015;36(1):119-130.

33. Trocha M, Merwid-Ląd A, Szuba A, Sozański T, Magdalan J, Szeląg A. Asymmetric dimethylarginine synthesis and degradation under physiological and pathological conditions. Adv Clin Exp Med. 2010; 19(2):223-243.

34. Vaghasiya JD, Sheth NR, Bhalodia YS, Jivani NP. Exaggerated liver injury induced by renal ischemia reperfusion in diabetes: Effect of exenatide. Saudi J Gastroenterol. 2010;16(3):174-180.

35. Toyoda-Akui M, Yokomori H, Kaneko F, et al. A case of drug-induced hepatic injury associated with sitagliptin. Intern Med. 2011;50(9): 1015-1020.

36. Gross BN, Cross LB, Foard J, Wood Y. Elevated hepatic enzymes potentially associated with sitagliptin. Ann Pharmacother. 2010;44(2): 394-395.

37. Raz I, Hanefel M, Xu L, Caria C, Williams-Herman D, Khatami H. Efficacy and safety of the dipeptidyl peptidase-4 inhibitor sitagliptin as monotherapy in patients with type 2 diabetes mellitus. Diabetologia. 2006;49(11):2564-2571.

38. Nauck MA, Meininger G, Sheng D, Terranella L, Stein PP. Efficacy and safety of the dipeptidyl peptidase-4 inhibitor, sitagliptin, compared with the sulfonylurea, glipizide, in patients with type 2 diabetes inadequately controlled on metformin alone: A randomized, doubleblind, non-inferiority trial. Diabetes Obes Metab. 2007;9(2):194-205. 